\title{
Updating Hearing Screening Practices in Early Childhood Settings
}

\author{
William D. Eiserman, PhD; Lenore Shisler, MS; \\ Terry Foust, AuD; Jan Bubrmann, PbD; \\ Randi Winston, AuD; Karl White, PhD
}

\begin{abstract}
Each day in the life of a young child with an undetected hearing loss is a day without full access to language. When hearing loss goes undetected, the resulting language deficits can become overwhelming obstacles to literacy, educational achievement, socialization, and school readiness. Several programs, such as Head Start, Early and Periodic Screening, Diagnosis and Treatment, and Part C of the Individuals with Disabilities Education Act, are responsible for providing hearing screening to many young children nationwide. These programs have typically had to rely on subjective hearing screening methods. Otoacoustic emissions technology, used widely in hospital-based newborn screening programs, is beginning to be recognized as a more practical and effective alternative when screening children from birth to 3 years of age. Successful otoacoustic emissions screening in early childhood settings is dependent on consultation from an experienced pediatric audiologist, selection of appropriate equipment, adherence to an appropriate screening and follow. up protocol, and access to training and follow-up technical assistance. When these elements are present, children with a wide range of hearing health conditions can be identified in a timely manner. Key words: deafness, hearing disorders, bearing loss, bearing screening, otoacoustic emissions, sensory screening
\end{abstract}

GEVERAL programs, such as Head Start, Early and Periodic Screening, Diagnosis and Treatment, and Part $C$ of the Individuals with Disabilities Education Act, are responsible for providing hearing screening to many young children nationwide. These programs have typically had to rely on subjective hearing screening methods including healthcare provider reports, informal observations of a child's behavioral response to sound, parent perceptions of a child's behavior, or prior

\footnotetext{
Autbor Affiliations: National Center of Hearing Assessment and Management, Utab State Unitersit): Logan (Drs Eiserman, Shisler; and White). Intermountain Healtb Care, Salt Lake Cit), Utab (Dr Foust): Department of Sociology: Illinois College, Jacksonville (Dr Bubrmann); and Ear Foundation of Arizona, Pboenix; (Dr Winston).
}

Corresponding Autbor: William D. Eiserman, PhD, National Center for Hearing Assessment and Management, 1880 Telluride In, Boulder CO 80305 (bearingbeadstart@aol.com). newborn hearing screening results (Munoz, 2003). Otoacoustic emissions (OAE) technology, used widely in hospital-based newborn screening programs and validated by professional organizations as an objective and reliable screening method (Joint Committee on Infant Hearing, 2007), is beginning to be recognized as a more practical and effective alternative when screening children from birth to 3 years of age.

\section{HOW MANY INFANTS AND YOUNG CHILDREN HAVE A HEARING LOSS?}

In the United States, approximately 1 of every 300 children is born with a permanent hearing loss, making it the most common birth defect in the country (White, 1996). Advances in technology have now made it possible to screen newborns for hearing loss and over the past 10 years, the percentage of infants screened at birth has increased from $3 \%$ to more than $95 \%$ (National Center for 
Hearing Assessment and Management, 2008). Although universal in concept, however, approximately $5 \%$ of newborns nationwide do not receive a hearing screening and less than $40 \%$ of the infants who refer from newborn screening are documented as having received the diagnostic assessment services they need (Centers for Disease Control and Prevention, 2005). In addition, not all hearing loss can be identified at birth because a child can lose his or her hearing at any point during early childhood. It is estimated that by the time children are in school, the cumulative incidence of severe permanent hearing loss is 6 per 1000 , including the 1 to 3 per 1000 likely to be detected at birth (Centers for Disease Control and Prevention, 2005; National Institute on Deafness and Other Communication Disorders, 2005). Finally, it is estimated that 35\% of preschoolers will have repeated episodes of ear infections, usually accompanied by a temporary hearing loss that can also disrupt the language learning process (American SpeechLanguage-Hearing Association, 2004). Hence, early childhood hearing screening programs are critical for identifying a range of hearing health conditions that can impede development for many children.

\section{WHAT ARE THE SHORTCOMINGS OF TRADITIONAL EARLY CHILDHOOD HEARING SCREENING METHODS?}

When physicians indicate that a child's hearing has been "checked," it usually means that the child's ear canal and tympanic membrane were examined using otoscopy and/or tympanometry or a behavioral observation was made of a child's response to sound (ie, bell-ringing or hand clapping). Although otoscopy and tympanometry are useful methods for identifying anatomical problems and middle ear disorders, such as otitis media, these methods cannot assess inner ear (cochlear) functioning. Informal behavioral observation of a child's response to sound, although intuitively attractive, is not a reliable method for assessing hearing loss in young children. This is due, in part, to the fact that children with significant hearing loss may have residual hearing that allows them to respond to certain sounds, while not necessarily allowing them to hear all speech sounds clearly. In addition, many naive screeners, including physicians, unintentionally provide visual cues at the same time as auditory ones. Children may therefore appear to be responding to sound when in reality they are taking their cues from visual prompts. Conversely, children with perfectly normal hearing may not respond to particular sounds simply because they are attending to other stimuli in the environment. These inconsistent responses to auditory prompts also make it unlikely for a child's hearing loss to be identified through parent questionnaires. Finally, referring to a child's newborn hearing screening results, which may have been obtained some months or years previously, cannot be assumed to be an accurate reflection of the child's current hearing status.

Unfortunately, by the time a child manifests delays in language, cognition, and social skills that trigger a parent or professional to request a full hearing evaluation, the delays are often so severe that a child may never be able to make up for lost language-learning time. The close connection between hearing, language acquisition, literacy, and school readiness demands that as technology improves, so also should the quality of early childhood hearing screening.

Like many head start programs serving children birth-to-three years of age, for years all we had been using were the bells, noise makers, and a parent questionnaire to screen the hearing of children in our program. We knew this was not adequate, but we didn't know what else we could be doing that would be a more objective method. Julie Quaid, Confederate Tribes of Warm Springs, American Indian Head Start, Warm Springs, Oregon, oral communication, March 13, 2002)

\section{WHAT IS O'TOACOUSTIC EMISSIONS SCREENING AND WHAT ADVANTAGES DOES IT OFFER?}

OAE technology is used widely in hospitalbased newborn hearing screening programs 


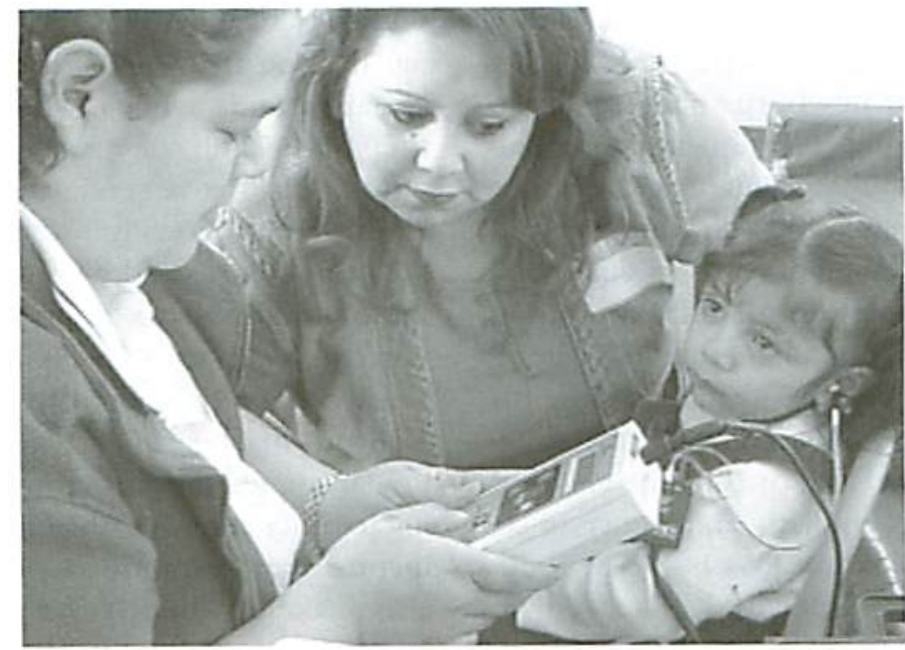

Figure 1. Photograph of child being screened using otoacoustic emissions technology.

and has been recognized by the Joint Committee on Infant Hearing. During OAE screening, the screener places a small probe, fitted with a sensitive microphone, into the child's ear canal (see Figure 1). The probe delivers a quiet sound into the ear. In a healthy ear, sound is transmitted through the middle ear to the inner ear where the outer hair cells of the cochlea respond by producing an emission sometimes described as an "echo." This emission travels back out through the middle ear and is then picked up by the microphone, analyzed by the screening unit, and a "pass" or "refer" result is displayed on the equipment screen. Every normal, healthy inner ear produces an emission that can be recorded in this way (Gorga et al., 1997).

The ear will not pass the screening if there is (a) a blockage in the ear canal, (b) a structural problem or excess fluid in the middle ear that interferes with hearing, or $(c)$ an impaired cochlea that is not responding normally to sound. Thus, OAE screening can help identify children who need to be evaluated for fluctuating loss associated with otitis media as well as those who may have permanent hearing loss. It is important to emphasize, however, that OAE screening is not an audiological diagnosis of hearing status. As with any type of hearing screening, children who do not pass the OAE screening should be referred for ap. propriate medical and audiological diagnosis and treatment.

OAE technology, when used with an appropriate protocol, holds great promise for screening children from birth to 3 years of age because it is

- objective and not dependent on a behavioral response from the child (can even be performed while the child is sleeping);

- painless;

- reliable and efficient (requiring about 5 minutes per child);

- hand-held and portable (suitable for screening in either center or home-based settings);

- simple to administer when a child initially enters an early childhood program, at annual intervals, and any other time parents or educators have concerns about the child's hearing health; and

- easy to use and does not require the screener to have advanced technical skill or in-depth understanding of the auditory system.

\section{IS OTOACOUSTIC EMISSIONS SCREENING FEASIBLE IN EARLY CHILDHOOD SETTINGS?}

In a recent study, the feasibility and effectiveness of screening young children using 
OAE technology was examined (Eiserman et al., 2007). In this study, 3486 children from birth to 3 years of age from 52 different Head Start Program sites were screened by Head Start staff, using OAE screening technology and an accompanying multi-step screening protocol. Of the 3486 children screened, a total of $183(5 \%)$ were referred for medical or audiological follow-up. Of these 183 children, 80 were identified with a hearing loss or disorder, requiring treatment or monitoring. Six of 80 were diagnosed as having permanent hearing loss, 63 were identified with otitis media (11 of whom were further diagnosed to have fluctuating conductive hearing loss associated with chronic middle ear infection), 2 were treated for occluded pressure equalization tubes, and 9 were treated for excessive earwax or congestion. This study suggests that OAE screening in early childhood settings helps identify approximately 1 of every 43 children as needing audiological treatment or monitoring and 1 of every 600 as having a permanent hearing loss that was not previously identified.

It is also important to note that staff involved in OAE screening implementation reported positive perceptions of their experience.

We have always assumed that the medical providers were screening for hearing during well-child visits. In fact, we relied on them for this. We have discovered that even though a child's medical record may indicate that ears have been checked, this does not necessarily mean a hearing screening has been performed. By implementing OAE hearing screening as a part of the battery of screenings we provide to all children, we are providing a valuable service that is seldom provided by anyone else. (Jyl Bosone, Mid-Columbia Children's Council, Hood River, Oregon, oral communication, July 10, 2004)

I screened a child who never passed after multiple attempts. He was referred to an audiologist and was eventually identified with a permanent hearing loss. When I got this news, I had mixed emotions. On the one hand, I felt sad for what this potentially means for the child and family. On the other hand, I was thrilled that we got this child in to see the audiologist to get tested and identified as early in his life as we did. He had had nearly a full year of disrupted language acquisition because of his hearing loss, but now he is going to have accommodations made so that he is not at a disadvantage because of his hearing loss. That's the whole point of identifying children with hearing losses as early as possible. I am very excited to be a part of this because it really does change lives. (Alissa Weller, Bear River Head Start, Logan, Utah, oral communication, July $10,2004)$

Overall, participants indicated that they preferred OAE screening over previous hearing screening methods because it

- is quick, reliable, and incurs no additional personnel costs;

- can be performed in a variety of natural environments, including settings where other children are playing and vocalizing at a moderate level;

- builds confidence that their hearing screening is based on accepted audiological practices;

- expedites the referral process for followup, promoting prompt attention from healthcare providers;

- contributes significantly to a child's medical home health record because OAE screening is seldom conducted in healthcare clinic or office settings; and

- serves as a model for early childhood hearing screening in other settings such as Part $\mathrm{C}$ programs, community health clinics, and private healthcare practices.

\section{WHAT ARE THE KEY ELEMENTS WHEN IMPLEMENTING AN OTOACOUSTIC EMISSIONS SCREENING PROGRAM?}

The feasibility study answered important questions about how OAE technology can be used effectively by early childhood staff to screen children from birth to 3 years of age. The results do not suggest, however, that all programs should purchase a piece of equipment and initiate screening independently. Successful implementation of an OAE screening program is dependent on several key elements: (a) involving a pediatric audiologist; (b) selecting OAE equipment; (c) adhering to 
a hearing screening protocol; and $(d)$ accessing training and technical assistance.

\section{Involving a pediatric audiologist}

Although OAE hearing screening does not need to be conducted by an audiologist, the involvement of a pediatric audiologist is essential in selecting OAE screening equipment, developing and implementing an appropriate screening and follow-up protocol, and providing training and technical assistance to screeners and other professionals conducting follow-up. If the program does not already have an established relationship with a pediatric audiologist, each state's Early Hearing Detection and Intervention Program (see www.infanthearing.org for state-specific contact information) can be a valuable resource for identifying a local pediatric audiologist as well as other professionals who can assist with OAE screening program implementation.

During the feasibility study, several Head Start programs were identified that had attempted to implement an OAE screening program without the involvement of a pediatric audiologist. One program had selected OAE equipment that was not appropriate for screening toddlers in natural settings, and staff erroneously concluded that OAE screening was not a viable method. Another program had failed to conduct an OAE rescreen of children referred to and treated by healthcare providers for middle ear conditions. This meant that the child's inner ear functioningthe part of the ear most commonly associated with permanent hearing loss-had never been screened. Several programs that had attempted to independently implement $\mathrm{OAE}$ screening demonstrated poor screening practices that resulted in high false-positive referral rates, unnecessarily long screening times, and overall frustration with the OAE screening method. If pediatric audiologists had been involved in providing training and technical assistance to staff, frustration may have been minimized and more accurate screening outcomes achieved.

\section{Selecting otoacoustic emissions equipment}

In conjunction with specific advice that pediatric audiologists have to offer, the following are general criteria to consider when selecting $\mathrm{OAE}$ equipment for screening children from birth to 3 years of age in natural settings:

- Portability: Equipment should be handheld, battery operated, and have a carrying case that enables the equipment to be easily stowed and moved from one setting to another.

- Capability: Equipment should be capable of screening a child's ear in approximately 2 minutes or less, be easy to operate, and feature only the essential capabilities needed for an OAE screening program. For example, it should provide visual feedback that tells a screener what to do if screening is not proceeding. It should display results in simple terms, such as "pass" or "refer," that require no interpretation. It is also important to select equipment that has been demonstrated to work well with toddlers and young children in addition to newborns. Some equipment works well in nursery settings where infants are sleeping but does not perform equally well with young children who are upright, awake, and physically active.

- Probe and probe tips: The probe and probe tips should be designed to stay seated snugly in the ear canal. The cord from the probe to the screening unit should be about 4 feet in length to reach from the child's ear to where the screening unit rests. A clip should be provided to secure the cord to the child's clothing during screening. The cost of disposable probe covers must also be taken into consideration.

- Customer support: Equipment vendors should provide hands-on training and materials related to equipment functions. They should also offer an adequate warranty that provides quick, reliable repair of equipment and loaner equipment 
when repairs are needed. Some vendors allow programs to try out screening units for up to 6 months prior to purchase.

- Purchase and maintenance costs: The cost of equipment should be less than $\$ 4000$ and include an initial set of probe tips and a replacement probe. It is important to consider the need for and expense of periodic equipment calibration.

\section{Adhering to a hearing screening protocol}

It is vital that a screening and follow-up protocol, administered under the supervision of a pediatric audiologist, be utilized to $(a)$ maximize the identification of children having permanent hearing loss as well as chronic middle ear disorders or other hearing health conditions; and (b) minimize overreferral of children for medical or audiological follow-up who did not actually need treatment ("falsepositive" referrals). Thus, the protocol should ensure that children with hearing health conditions would be identified while being practical for program staff, physicians, and audiologists to implement. Before starting an OAE screening program, it is wise to examine what screening practices, if any, are already in place and how OAE screening will replace or be combined with these practices. For example, parent questionnaires can continue to be administered as part of an OAE screening program if desired.

Key components of the recommended protocol used in the feasibility study are summarized below (Figure 2):

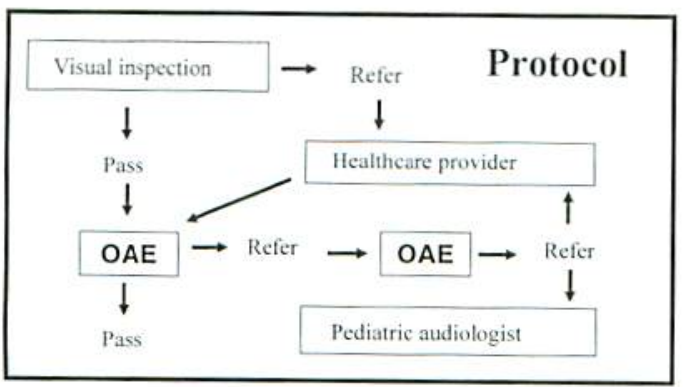

Figure 2. Key elements of a recommended otoacoustic emissions (OAE) screening protocol.
1. The first step is for the screener to complete a visual inspection of each ear. This includes examining the outer ear for $a b$ normalities, foreign objects or blockage in the car canal, any fluids draining from the ear, or noticeable odor. If any abnormal conditions are present, the child is referred to a healthcare provider for a medical examination.

2. If the child passes the visual inspection, or on receiving medical clearance from a healthcare provider after an earlier referral, the OAE screening is conducted on each ear. If both ears pass the test, the child's hearing screening is considered complete.

3. If the child does not pass the screening on both ears, or if the screener is unable to complete the screening (generally because the child is uncooperative or the environment is too noisy) the screening is repeated on a subsequent day. Any ear not passing the initial screening is rescreened within 2 weeks. If a passing result is not achieved on both ears, the child is referred to a healthcare provider for medical assessment/intervention for a possible middle ear disorder.

4. After treatment and/or medical clearance is obtained, the OAE screening process is repeated on any ear that did not pass the test on earlier screening attempts. If the ear passes the test, no further testing is needed until the next scheduled screening. However, if the ear does not pass the test after medical clearance, the child is referred to a pediatric audiologist for evaluation.

Although screeners are trained to follow the standard protocol, they must exercise their own judgment when individual circumstances warrant more immediate referral to a healthcare provider or audiologist.

Head Start staff participating in the feasibility study commented on the practicality of using $\mathrm{OAE}$ technology to screen young children.

Initially, I was concerned about the additional time and costs that might be associated with 
implementing an OAE screening program. In reality, OAE screenings are quicker to perform than our previous method, taking about 3 to 5 minutes per child. We do have to rescreen some of the children, however, which has taken some strategizing, but we simply rearranged a few activities to get this accomplished and haven't encountered any prohibitive barriers in terms of time or money. Now I can't imagine using any other method. (Barbara Williams, Washington State Migrant Council, Sunnyside, Washington, oral communication, July 6 , 2004)

One of the positive outcomes of doing OAE screenings is that physicians are responding to my referrals. I used to refer a child for hearing simply because they didn't respond to my clapping. Now that I have an objective screening tool, the physicians are able to follow through. The physicians are seeing us in a new light - as more legitimate partners in meeting the health needs of the children we serve. As a consequence, we have played an important role in identifying numerous children with fluctuating hearing losses caused by untreated middle ear infections and one child with a permanent hearing loss. (Sara Rolfs, Chelan-Douglas Child Services, Early Head Start, Wenatchee, Washington, oral communication, June 20,2004 )

\section{Access to training and technical assistance}

Although anyone skilled in working with children can learn to conduct OAE screening, program staff will need thorough training and follow-up technical assistance. In the feasibility study, 6-hour training workshops were provided to program staff that included the following components:

- Large-group instruction in which a training team of 3 to 4 experienced screeners, including 1 pediatric audiologist, provided general information to the entire group of 12 to 16 screener trainees.

- Small-group instruction in which each trainer assisted 3 to 4 screener trainees in learning to use the equipment by screening one another. Trainers then supervised trainees in small-group hands-on sessions as each participant screened at least 5 children and documented screening results.
- Audio-visual and written materials used to standardize the training were also given to participants for review purposes.

The most common needs for technical assistance during the initial feasibility study related to care and maintenance of equipment, screening techniques, case-specific consultation, adherence with the protocol, training because of staff turnover, and resources for communicating with the medical community.

Learning to implement OAE screening and followup practices is relatively simple. By the end of a one-day training, we were off and running, and have needed only a little bit of help since we got started. Training was the key for us. Learning to do OAE screening is not difficult, but it is just tricky enough that training is important. I don't know that we would have ever gotten this going without the training we received. (David Bennett, Oregon Child Development Coalition, Migrant Head Start, Ashland, Oregon, oral communication, May 16, 2004)

\section{CONCLUSIONS}

Each day in the life of a young child with an undetected hearing loss is a day without full access to language. When hearing loss goes undetected, the resulting language deficits can become overwhelming obstacles to literacy, educational achievement, socialization, and school readiness (Moeller, 2000). Before OAE technology was available, early childhood educators and healthcare providers had no choice but to rely on informal behavioral observations, checklists, and questionnaires to screen for hearing loss. Fortunately, OAE screening technology has proven to be a reliable screening method, used extensively by hospitals implementing newborn hearing screening programs, and most recently, by a number of Head Start programs serving children from birth to 3 years of age. Successful OAE screening in early childhood settings is dependent on consultation from an experienced pediatric audiologist, selection of appropriate equipment, adherence to an appropriate screening and follow-up protocol, and access to training and follow-up technical 
assistance. When these elements are present, children with a wide range of hearing health conditions can be identified in a timely manner. Medical, audiological, and/or educational interventions can then be provided to dramatically minimize or eliminate the long-term developmental consequences commonly associated with hearing loss.

\section{REFERENCES}

American Speech-Language-Hearing Association. (2004) Even minimal, undetected bearing loss burts academic performance, research sbous. Retrieved May 10, 2008, from http://wwwasha.org/about/news/ 2004/04ConvMinHrngLoss.htm

Centers for Disease Control and Prevention. (2005). Preliminary summary of 2005 EHDI data, infants test for bearing loss-united states, 1999-2001. Retrieved March 17, 2008, from http://www.cdc.gov/ ncbddd/chdi/documents/Nat_Summ_2005_Web_V5. pdf

Eiserman, W., Shisler, L., Foust, T., Buhrmann, J., Winston, R, \& White, K. (2007). Screening for hearing loss in early childhood programs. Early. Cbildbood Research Quarterly, 22(1), 105117.

Gorga, M. P., Neely, S. T., Ohlrich, B., Hoover, B., Redner, J. \& Peters, J. (1997). From laboratory to clinic: A large scale study of distortion product otoacoustic emissions in ears with normal hearing and cars with hearing loss. Ear and Hearing, 18(6), 440455.

Joint Committee on Infant Hearing. (2007). Joint committee on infant hearing, year 2007 position state. ment: Principles and guidelines for early detection and intervention programs. Pediatrics, 120(4), 898921.

Mocller, M. P. (2000). Early intervention and language development in children who are deaf and hard of hearing. Pediatrics, 106(3), E43.

Munoz, K. (2003). Survey of current bearing screening practices in early Head Start, American Indian Head Start and Migrant Head Start programs. Logan: National Center for Hearing Assessment and Management, Utah State University.

National Institute on Deafness and Other Communication Disorders. (2005). NIDCD outcomes researcb in cbildren and bearing loss, statistical report: prevalence of bearing loss in us children. Retrieved March 27. 2008, from http://www.nided.nih.gov/funding/ programs/hb/outcomes/report.html

National Center for Hearing Assessment and Management. (2008). Unitersal newborn bearing screening: Summary statistics of UNHS in the united states. Retrieved March 12, 2008, from http://www. infanthearing.org/status/unhsstate.html

White, K. R. (1996). Universal newborn hearing screening using transient evoked otoacoustic emissions: Past, present, and future. Seminars in Hearing, 17(2), 171-183. 